\title{
Local Directional Cues Control Growth Polarity of Dopaminergic Axons Along the Rostrocaudal Axis
}

\author{
Shin-ichiro Nakamura, ${ }^{1}$ Yasuko Ito, ${ }^{1}$ Ryuichi Shirasaki, ${ }^{1,2}$ and Fujio Murakami ${ }^{1,2,3}$ \\ ${ }^{1}$ Laboratory of Neuroscience, Division of Biophysical Engineering, Graduate School of Engineering Science, and ${ }^{2}$ Core \\ Research for Evolutional Science and Technology/Murakami Laboratory, Center for Advanced Research Projects, Osaka \\ University, Machikaneyama 1-3, Toyonaka 560-8531, Japan, and ${ }^{3}$ Division of Behavior and Neurobiology, National \\ Institute for Basic Biology, Myodaiji-cho, Okazaki 444-8585, Japan
}

The vertebrate CNS is composed of a variety of longitudinal axonal tracts extending rostrally and caudally. Although recent studies have demonstrated that chemoattraction and chemorepulsion play key roles in axon guidance along the circumferential axis in the neural tube of the vertebrate, mechanisms of axonal elongation along the longitudinal axis, and most importantly, what determines rostrocaudal polarity of axonal growth, remains unknown. Here, we examined the mechanism that guides midbrain dopaminergic axons rostrally, using flat wholemount preparations of embryonic rat brain both in vivo and in vitro.

At embryonic day 11 (E11) and early stage E12, dopaminergic neurons in the ventral midbrain extended short axons dorsally. By middle stage E12, these axons had increased in number, some deflecting rostrally and others caudally. At E13, almost all axons showed rostrally oriented growth heading toward the forebrain targets. In in vitro whole-mount preparations prepared from an E12 embryo and cultured for $24 \mathrm{hr}$, these axons showed rostrally oriented growth, but when they were forced to grow on substratum of reversed rostrocaudal polarity, they turned abruptly and grew following the polarity of the reversed midbrain substratum. These results suggest that local directional cues in the midbrain guide these axons rostrally and support the idea that substratum-associated polarized cues play an important role in axon guidance along the longitudinal axis.

Key words: rat embryo; whole-mount culture; local directional cue; dopaminergic neuron; tyrosine hydroxylase (TH); polarity; longitudinal axis
The vertebrate neural tube is composed of an orthogonal array of axons extending along the longitudinal and circumferential axes. A fundamental issue in developmental neuroscience is to clarify the mechanisms that guide growth cones along these two axes.

Recent studies have unraveled important mechanisms contributing to the guidance of axons along the circumferential axis (Colamarino and Tessier-Lavigne, 1995a; Murakami and Shirasaki, 1997). Commissural axons originating from the alar plate in the spinal cord through to the midbrain are attracted (Tessier-Lavigne et al., 1988; Shirasaki et al., 1995, 1996; Tamada et al., 1995), whereas some axons from the basal plate are repelled by the floor plate at a distance in vitro (Colamarino and TessierLavigne, 1995b; Guthrie and Pini, 1995; Tamada et al., 1995; Shirasaki et al., 1996), suggesting that floor plate chemoattraction and chemorepulsion contribute to the guidance of circumferential axons.

Another major component that comprises the axonal tracts of the neural tube is longitudinal axons. Although it is of great importance to clarify factors controlling the growth polarity of axons along the longitudinal axis, surprisingly little is known

Received Oct. 8, 1999; revised March 9, 2000; accepted March 9, 2000.

This work was supported by a grant for priority areas (07279101) from the Ministry of Education, Science, Sports, and Culture of Japan and Core Research for Evolutional Science and Technology. We thank E. S. Ruthazer, N. Yamamoto, A. Tamada, Y. Oda, K. Kobayashi, and N. Matsushita for critical reading of this manuscript and Dr. W. B. Stallcup for his gift of F84.1 antibody.

Correspondence should be addressed to Fujio Murakami, Laboratory of Neuroscience, Division of Biophysical Engineering, Graduate School of Engineering Science, Osaka University Machikaneyama 1-3, Toyonaka, Osaka 560-8531, Japan. E-mail: murakami@bpe.es.osaka-u.ac.jp.

Copyright (C) 2000 Society for Neuroscience $\quad 0270-6474 / 00 / 204112-08 \$ 15.00 / 0$ about this process. One possible mechanism that comes from an analogy with our knowledge on the guidance of circumferential axons is that axon guidance along the rostrocaudal axis is also regulated by some long-range diff usible cues. However, to date no evidence has been provided to support this view. The fact that the neural tube is polarized along the anteroposterior as well as the dorsoventral axes (Kelley and Melton, 1995; Lumsden and Krumlauf, 1996) raises another intriguing possibility that rostrocaudal polarity of the neural tube is in some way involved in axon guidance along the longitudinal axis. However, in previous studies, rotation of neural tube tissue along the rostrocaudal axis in early development did not reverse the growth polarity of longitudinal axons (Hibbard, 1965; Holder et al., 1987; Yaginuma and Oppenheim, 1991; Matsuno and Nakamura, 1993; Nordlander and Liu, 1996), which fails to support the notion that the neural tube is polarized along the rostrocaudal axis in terms of axon guidance cues. Interpretation of these experiments, however, may be complicated by the possibility that the transplanted brain or spinal cord was repolarized because of signals from host tissues.

To unravel mechanisms that determine the growth polarity of axons along the rostrocaudal axis minimizing the effect from surrounding tissues, we have developed a whole-mount in vitro preparation of embryonic rat brain and examined development of midbrain dopaminergic neurons. These neurons develop in the ventral midbrain and extend axons rostrally to innervate the striatum, limbic system, and neocortex (Lindall and Björklund, 1983), providing an excellent opportunity of studying axon guidance along the longitudinal axis. We show that the neural tube is polarized rostrocaudally in terms of axon guidance cues and 
suggest that local polarized cues in the midbrain substratum determine growth polarity of these axons.

\section{MATERIALS AND METHODS}

\section{Animals}

The brains of embryonic day 11 (E11)-E13 Wistar rats were used to examine the development of tyrosine hydroxylase-positive $\left(\mathrm{TH}^{+}\right)$axons in the midbrain because $\mathrm{TH}^{+}$dopaminergic neurons in the midbrain begin to differentiate during this period. Because development of the dopaminergic projection proceeds rapidly, E12 rat embryos dissected out at three different times of the day were analyzed. These were 6:00 A.M., 12:00 P.M. (noon), and 6:00 P.M., and were termed early (E12-e), middle (E12-m), and late (E12-1) stage, respectively. The embryonic brains were cut along the dorsal and ventral midlines, and the regions rostral to the eye vesicle and caudal to the hindbrain were removed. Hemibrain thus obtained was flattened and fixed with $4 \%$ paraformaldehyde. The tissue was stained with an antibody against $\mathrm{TH}$ (polyclonal antibody; 1:250; Chemicon, Temecula, CA) and Cy3-conjugated secondary antibody (1: 250; Jackson ImmunoResearch, West Grove, PA), as described previously (Shirasaki et al., 1998). In some cases, it was also stained with F84.1 (1:20; a gift from Dr. W. B. Stallcup) followed by FITC-conjugated secondary antibody (1:150; Vector Laboratories, Burlingame, CA). No staining was observed when normal rabbit serum was applied in place of anti-TH antibody.

\section{Culture}

Culture on collagen-coated membrane. Similarly prepared brain tissues taken from E12-m rat, were cultured on a collagen-coated membrane (Yamamoto et al., 1989) (Transwell Collagen, Corning Costar, Cambridge, MA; catalog \#3492). The explants were placed on the membrane with the ventricular side of the explants facing down and cultured for 24 $\mathrm{hr}$ as previously described (Shirasaki et al., 1996), except that hormone supplements (except for transferrin and insulin) were not added into the culture medium. To facilitate attachment of the tissue to the membrane, the level of culture medium was adjusted to the upper surface of the tissue during the initial $7 \mathrm{hr}$ and thereafter, culture medium was supplemented to submerge the explants. Two types of coculture were performed. The first one was a coculture of an explant dissected from the ventral one-third of the midbrain that included $\mathrm{TH}^{+}$neurons and the dorsal two thirds of the midbrain excised from the other side of the neural tube. The second type was a coculture of three midbrain pieces. The dorsal midbrain tissue from the other side of the neural tube was cut into two pieces along the dorsoventral axis. Two caudal halves thus prepared from two littermates were put together and juxtaposed to a ventral midbrain explant. In some experiments, the rostral and caudal halves were transposed along the rostrocaudal axis to culture with the ventral midbrain. After culturing, the explants were fixed with $4 \%$ paraformaldehyde for $6 \mathrm{hr}$ and washed with PBS. Whole-mount immunohistochemistry was then performed on the culture explants after detaching the explants from the membrane filter. The staining procedures are described above. To ensure that $\mathrm{TH}^{+}$axons had not grown rostrally at the start of the culture, the ventral midbrain on the other side of the brain was fixed and stained for TH without culturing (see Fig. $2 A$ ). E13 brain was also used in some experiments, which provided similar results as E12 preparations.

Culture in collagen gels. The ventral one-third of the midbrain that contains $\mathrm{TH}^{+}$neurons and the midbrain-hindbrain boundary region were dissected out from E13 rats. The floor plate was carefully excluded from the explant, because it was shown to repel $\mathrm{TH}^{+}$axons (Tamada et al., 1995). These explants were embedded in collagen gels and cultured for $2 \mathrm{~d}$ as previously described (Shirasaki et al., 1996). After fixation, the cultured explants in collagen gels were immunostained using anti-TH antibody as described above.

\section{Quantification}

The angle of axonal growth was measured by referring to the border between the ventralmost region of the mesencephalon (VM) and dorsal mesencephalon (DM) explants, using NIH Image aided by software developed by E. Ruthazer. The mean of the angles of the connecting lines between the tips of the three longest $\mathrm{TH}^{+}$axons, the midpoint of the rostrocaudal border $\mathrm{VM}-\mathrm{DM}$ region intersected by $\mathrm{TH}^{+}$axons, and the caudal end of the border were calculated for each culture. Averaging the angles of the three longest axons were found to well represent the angle of the $\mathrm{TH}^{+}$axon bundle.
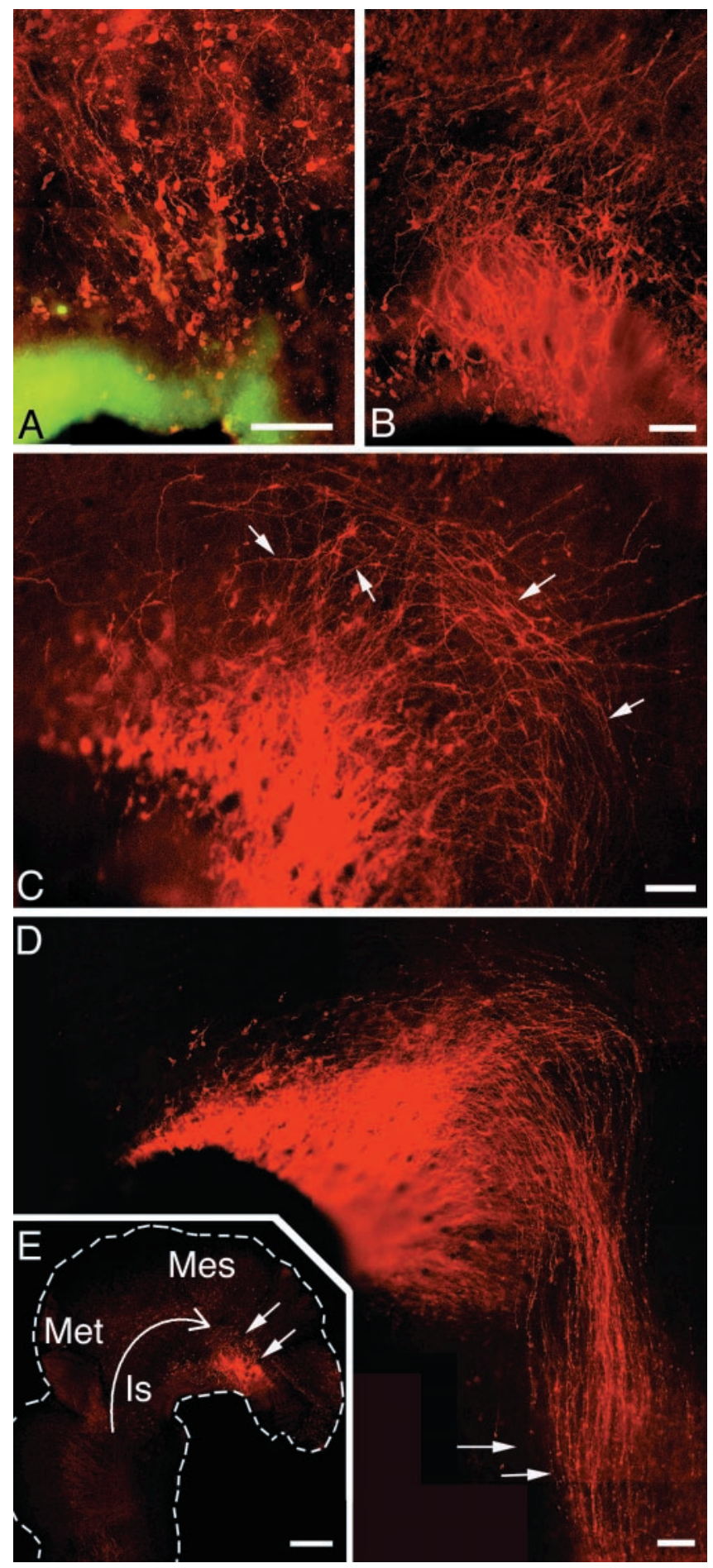

Figure 1. Development of midbrain $\mathrm{TH}^{+}$axons in a flat-mounted hemibrain preparation in vivo. A, At the early stage of E12 (E12-e), a small number of $\mathrm{TH}^{+}$neurons (stained in red) were found to extend short axons. $B$, At the middle stage of E12 (E12-m), $\mathrm{TH}^{+}$neurons extended axons dorsally without notable directed growth along the rostrocaudal axis. $C$, At late stage of E12 (E12-1), axons begin to show clear directed rostral growth (arrows). D, At E13 a substantial proportion of axons arrived at the diencephalon (arrows). In $A$, the floor plate was stained with F84.1 antibody (stained in green) to show the rostrocaudal axis and the ventral midline region of the preparation. $E$, A low-magnification view of a flat-mounted preparation from an E12-m rat embryo shown in B. Curved arrow indicates rostral and rostrocaudal axis, and arrows indicate a region of $\mathrm{TH}^{+}$neurons. Mes, Mesencephalon; Met, metencephalon; Is, isthmus. Scale bars: $A-D, 100 \mu \mathrm{m} ; E, 300 \mu \mathrm{m}$. 

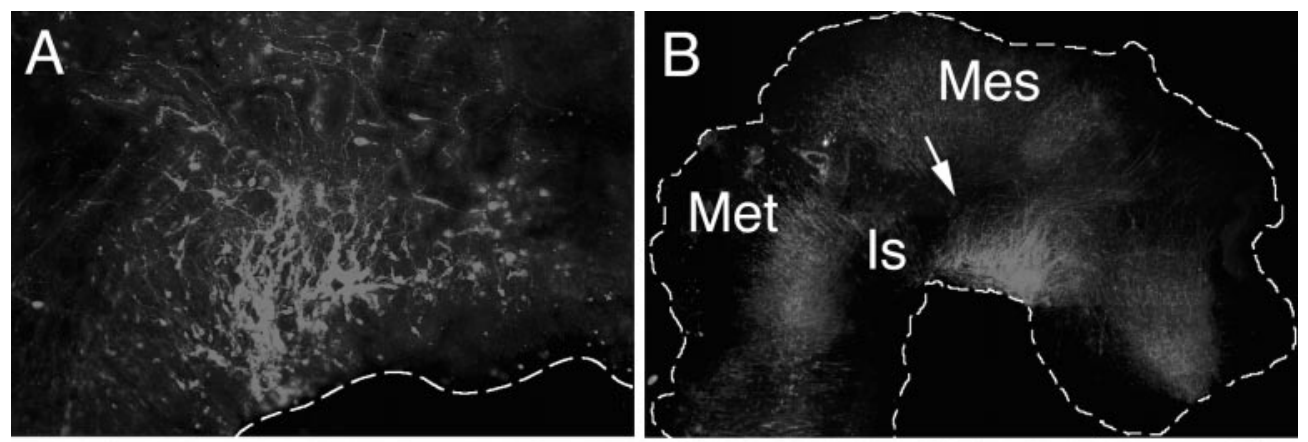

Figure 2. Development of midbrain $\mathrm{TH}^{+}$axons in vitro. $A, \mathrm{TH}^{+}$axons before the start of culture. The left half of the midbrain was immersed in a fixative without culturing and stained for $\mathrm{TH}$. Note that $\mathrm{TH}^{+}$axons had not shown rostrally oriented growth at this stage of development, middle stage of E12. B, Lower magnification view of the hemibrain used for culture. Arrow indicates the location of $\mathrm{TH}^{+}$neurons. $C$, Brains at the middle stage of E12 were cultured for $24 \mathrm{hr}$. $\mathrm{TH}^{+}$axons leaving the ventral midbrain region start to grow rostrally (arrows), although some axons deflect dorsally. The right half of the embryonic rat brain was used. Dashed line indicates tissue border. Scale bar, $100 \mu \mathrm{m}$.

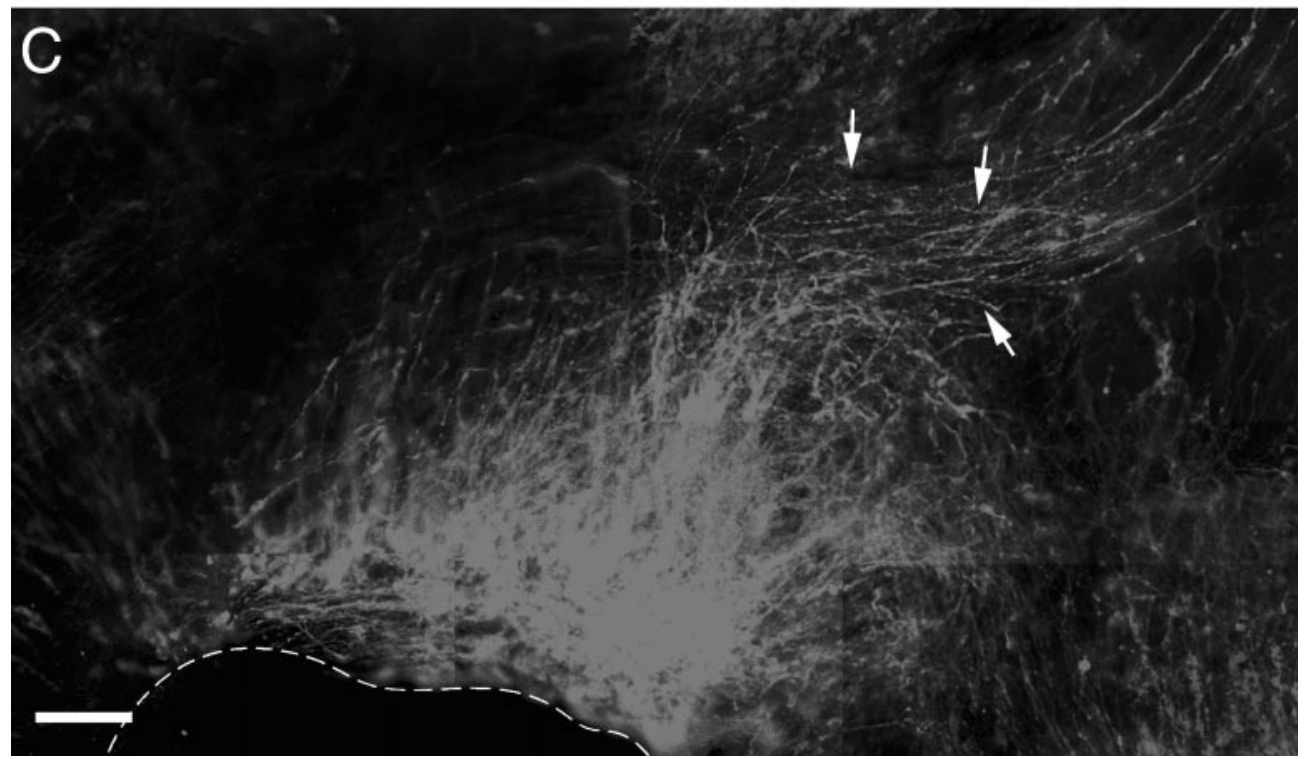

For quantification of axons in two DM explants cultured with a VM explant, a straight line was drawn from the DM explant border on dorsal edge to that of ventral edge, where the border was easily recognized from its rugged contours. Then distances between all discernible $\mathrm{TH}^{+}$axon tips and the line were scored. $\mathrm{TH}^{+}$axons occasionally elongated around the border between VM and DM. Such axons were excluded from the analysis. Axons growing directly from VM to rostrally placed half of DM explant without crossing the border were also excluded from the analysis.

\section{RESULTS}

\section{Development of midbrain dopaminergic axons in rat whole-mount preparation}

We first studied the early development of midbrain dopaminergic axons in the rat embryo, by immunostaining with an antibody against tyrosine hydroxylase $(\mathrm{TH})$, the rate-limiting enzyme in dopamine synthesis. At E11 and E12-e, a small number of $\mathrm{TH}^{+}$ neurons were found in the ventrorostral mesencephalon, both close to and inside the floor plate, extending short axons dorsally (Fig. $1 A$ ). By E12-m, $\mathrm{TH}^{+}$axons had increased in number, some deflecting rostrally and others caudally (Fig. 1B,E). At E12-1 the axons began to show clear rostrally oriented growth (Fig. $1 C$, arrows), although a substantial proportion of axons in the caudal part did not show a clear trend. By E13, however, almost all axons showed rostrally oriented growth heading toward the forebrain targets (Fig. 1D). Thus, $\mathrm{TH}^{+}$axons appear to start growing rostrally at around middle stage E12.

\section{Development of midbrain dopaminergic axons in culture}

We next developed a whole-mount culture preparation that can mimic the development of dopaminergic axons in vivo. We used embryos of E12-m, when $\mathrm{TH}^{+}$axons had not yet shown evident directional growth along the rostrocaudal axis (Figs. 1B, 2A). After $24 \mathrm{hr}$ in culture the preparations were fixed and stained for TH (Fig. $2 B$ ). In these preparations, $\mathrm{TH}^{+}$axons showed clear rostrally oriented growth toward the diencephalon (Fig. 2C). These results suggest that the mechanisms that guide $\mathrm{TH}^{+}$axons rostrally are retained in our culture preparations.

\section{Dopaminergic axons grow rostrally in the absence of the isthmus or diencephalon}

One possible mechanism that may explain the rostrally oriented growth of $\mathrm{TH}^{+}$axons is chemotactic guidance by gradients of diff usible molecules (Ramón y Cajal, 1892; for review, see Tessier-Lavigne, 1992). The finding that the midbrain-hindbrain boundary, the isthmic region, is a source of diff usible molecules that organizes the midbrain and the cerebellum (Bally-Cuif and Wassef, 1995; Crossley et al., 1996; Lee et al., 1997; Ye et al., 1998) raises the possibility that cells in this region secrete some diff usible molecule that repels $\mathrm{TH}^{+}$ axons rostrally. To test this possibility, the isthmic region was removed before the midbrain tissue was put into culture (Fig. $3 A$ ). Rostrally oriented growth of axons was not affected by this manipulation ( $n=10$ ) (Fig. $3 A, C)$, eliminating the notion of a chemotactic gradient diff using from the isthmus. Consistent with these results, $\mathrm{TH}^{+}$axons, emanating from a ventral midbrain explant in collagen gels, extended toward and even invaded into an isthmic explant placed at a distance $(n=4)$ (Fig. $3 D, E$ ). Similar to isthmus-deleted preparations, $\mathrm{TH}^{+}$ axons grew rostrally in diencephalon-deleted preparation $(n=$ 

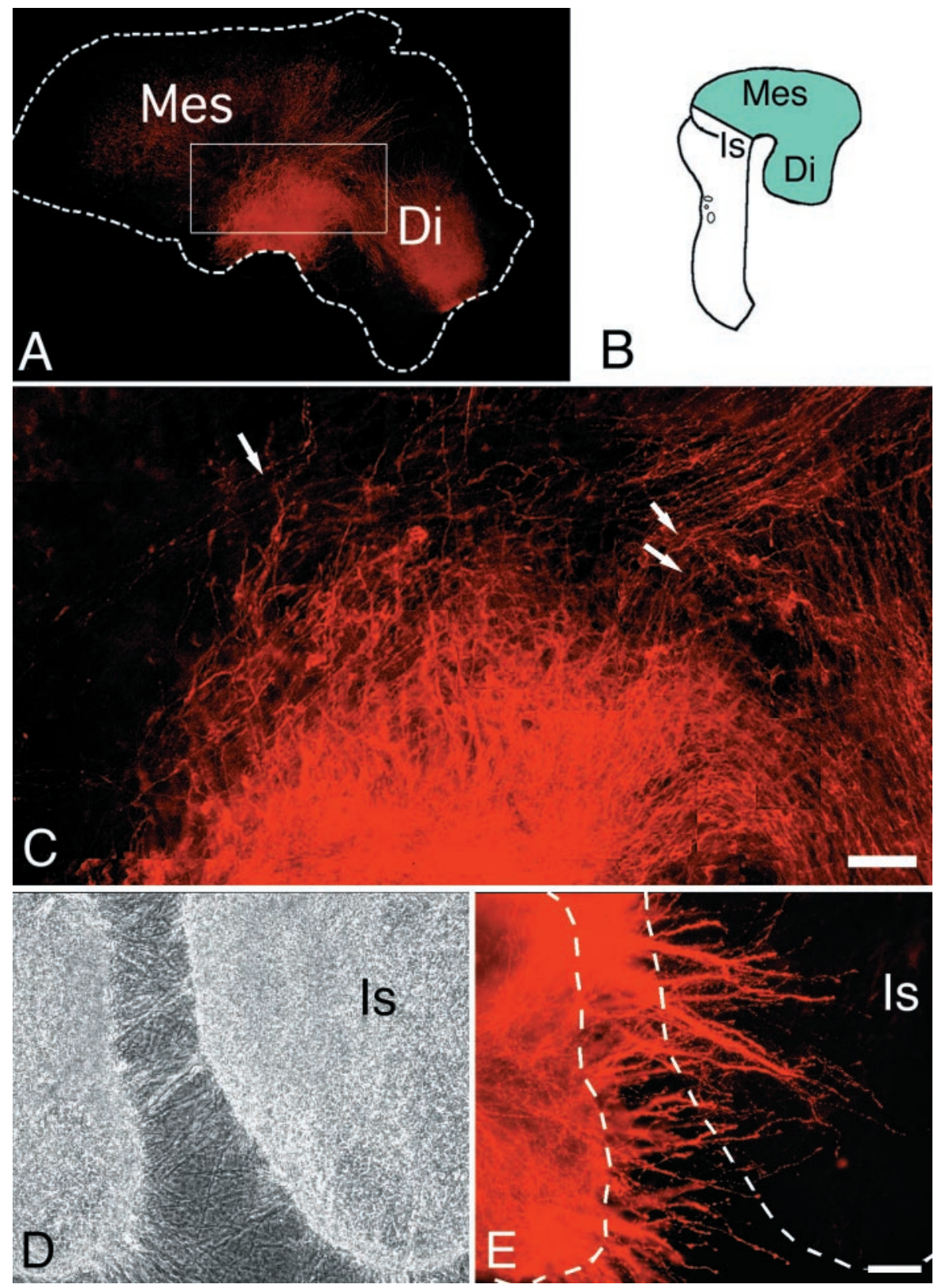

Figure 3. Development of midbrain $\mathrm{TH}^{+}$axons in isthmus-deleted preparation. $A$, Lower magnification view of the manipulated tissue. The region caudal to the isthmus was removed at the middle stage of E12, and flat-mounted hemibrain preparation was cultured for $24 \mathrm{hr}$. Rectangle represents the region shown in C. B, Schematic diagram showing the region used for culture (shown in blue). $C$, $\mathrm{TH}^{+}$axons leaving the ventral midbrain region extend rostrally (arrows) just as control preparations (Fig. 2). $D, E$, Coculture of ventral midbrain explant containing $\mathrm{TH}^{+}$axons and isthmic region $(I s)$ in collagen gel. Explant of the isthmus was carefully prepared to exclude the floor plate. A phase-contrast micrograph $(D)$ and corresponding immunofluorescent micrograph $(E)$. Note that $\mathrm{TH}^{+}$axons extend toward the isthmic explant. Mes, Mesencephalon; $D i$, diencephalon; Is, isthmus. Scale bars, $100 \mu \mathrm{m}$.
5) (data not shown, but see Fig. 4), indicating that chemoattraction by the diencephalon is also not responsible.

\section{Redirected growth of dopaminergic axons on midbrain substratum of reversed rostrocaudal polarity}

Another possible mechanism for rostrally oriented growth of the axons is that the substratum for axonal growth is in some way polarized along the rostrocaudal axis. Involvement of a substratum-associated polarizing cue has been repeatedly suggested for the guidance of sensory axons of the leg, wing, and antenna of insects (Bentley and Caudy, 1983; Berlot and Goodman, 1984). If this is the case, axons that enter a region of reversed rostrocaudal polarity should reorient their growth direction to follow the polarity of the altered substratum. To test this we removed the VM from one hemibrain, and juxtaposed it to the ventral side of the DM of the other half of the midbrain whose ventralmost region had also been removed (Fig. 4A), resulting in a reversal of rostrocaudal polarity of
VM against DM. As shown in Figure 4, $\mathrm{TH}^{+}$axons that initially grew rostrally (leftward in photo) in the VM transplant changed their growth direction after entering the DM, growing in the opposite direction in accordance with the rostrocaudal polarity of the DM (Fig. $4 B-D$ ). In control experiments in which DM and VM taken from the same side of the brain were put together in juxtaposition and cultured, $\mathrm{TH}^{+}$ axons showed normal rostrally oriented growth (Fig. 4E), indicating that the manipulation itself does not affect growth polarity of the axons. Quantification of these results agreed with our interpretation (Fig. $4 F, G$ ). The most straightforward explanation of the results is that $\mathrm{TH}^{+}$axons grow rostrally relying on directional cues associated with the substratum in the midbrain.

To further ensure that the directional cues are localized to midbrain region where $\mathrm{TH}^{+}$axons extend, DM explant was divided into dorsal and ventral halves, and VM explant from the 
Figure 4. Altered orientation of axonal growth on substratum with reversed rostrocaudal polarity. $A$, Schematic diagram showing regions of the brain used for the experiment. A piece of tissue was taken from the dorsal part of the right mesencephalon (RDM) (shown in red) and another piece from the ventralmost region of the left mesencephalon $(L V M)$ (shown in blue). These explants were put together $(B)$ and cultured for $24 \mathrm{hr}$. Note that rostral $(r)$ is to the left for LVM, whereas rostral is to the right for RDM. $c$, Caudal; $d$, dorsal; $v$, ventral. $C, \mathrm{TH}^{+}$axons extending from $\mathrm{LVM}$ and invading into RDM. Fluorescent micrograph of a corresponding view with $B . D$, High-magnification view of $C$. Axons starting to grow rostrodorsally in the LVM (see arrowheads) changed their growth direction at the border of explants (dashed lines) and started to grow after the rostrocaudal polarity of the RVM (small arrows). The border is discernible under bright-field illumination (B, black arrows). E, Control experiment in which DM and VM were taken from the same side of the brain. Unlike $D, \mathrm{TH}^{+}$axons continue to grow rostrally after crossing the explant border. For control experiments, the VM explant was placed back in its original position but slightly shifted caudally. This is because in our preliminary experiments in which VM was put back in its exact original position, axons arrived at the rostral edge of DM after extending for a short distance and then turned dorsally along the edge of the explant. In $D$ and $E$, horizontal arrows point to rostral. Scale bars: $B, C$, $200 \mu \mathrm{m} ; D, E, 100 \mu \mathrm{m} . F$ illustrates the method of axon angle measurement (see Materials and Methods for details). $G$, Quantification of the angle of $\mathrm{TH}^{+}$axons entering into dorsal mesencephalon with reversed rostrocaudal polarity. Ordinate: number of explant cultures. Abscissa: turning angle of axons. Green columns show data from control experiments in which VM and DM explants were taken from the same side of the neural tube, whereas blue columns show those from experiments in which the left VM explants were cultured with the right DM.
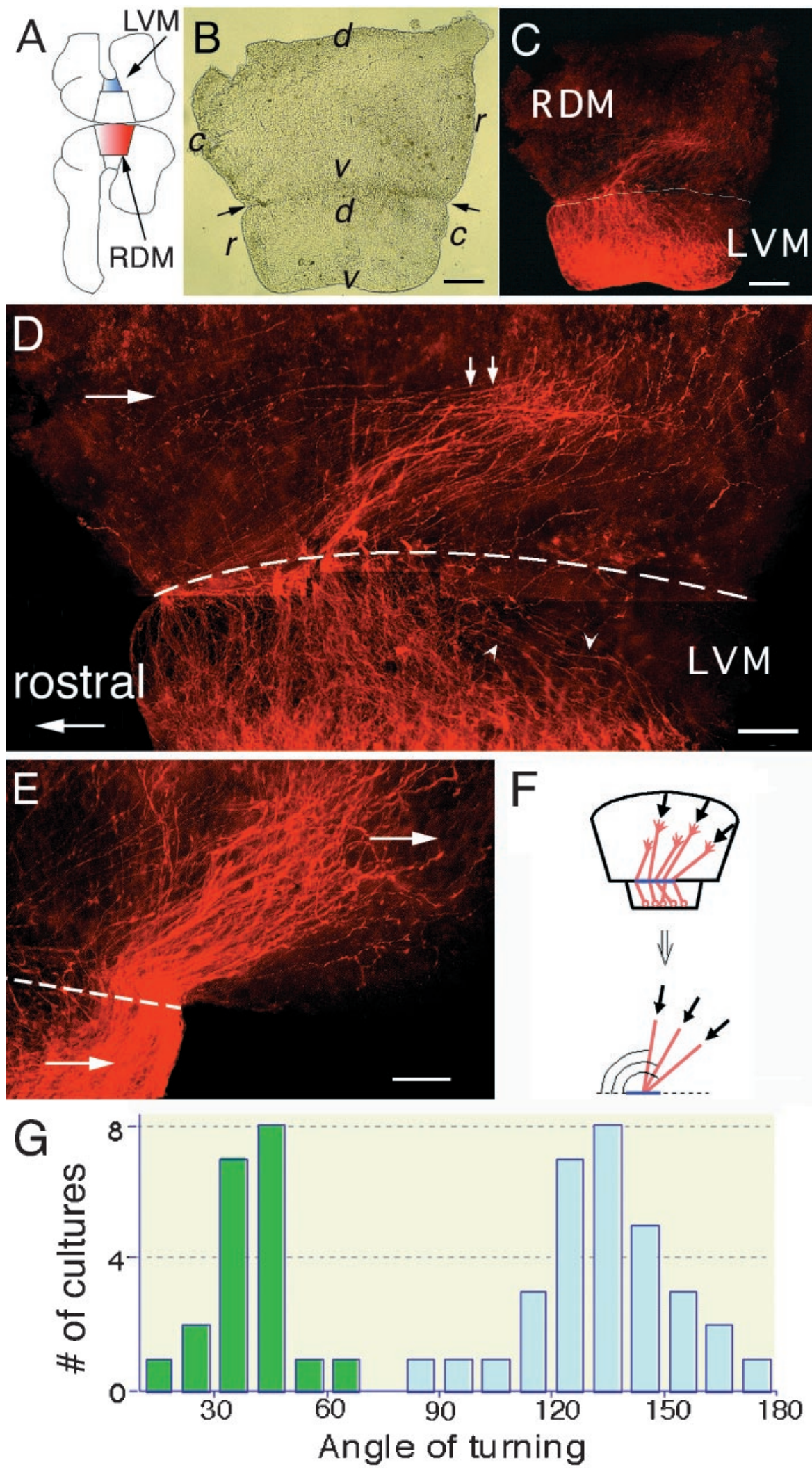

other side of the neural tube was juxtaposed to the ventral half (Fig. 5, inset). As shown in Figure 5, $\mathrm{TH}^{+}$axons extended following the polarity of the ventral DM explant, clearly demonstrating localized directional cues.

\section{Gradient of midbrain substratum may guide dopaminergic axons rostrally}

The polarity of tissues could be generated by a homogeneously distributed cue or a gradient of a substrate-associated cue (Palka, 


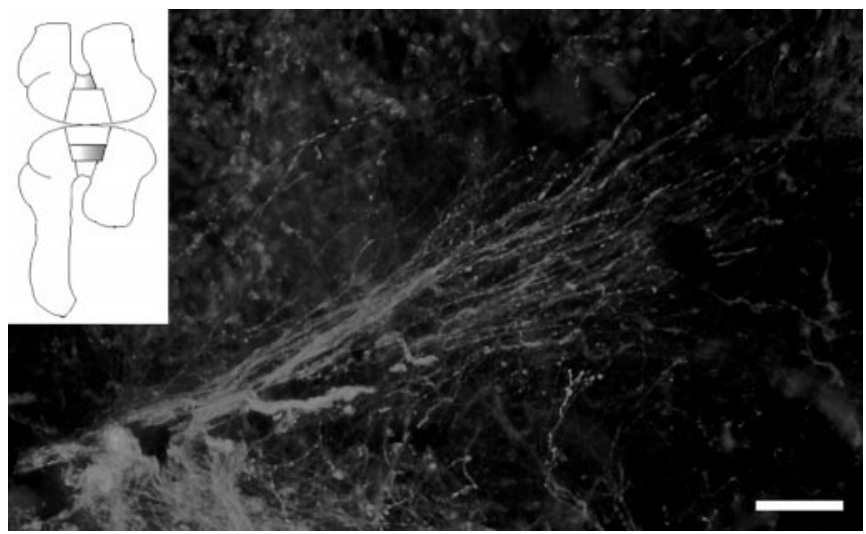

Figure 5. Evidence that localized directional cues in midbrain substrate guide $\mathrm{TH}^{+}$axons rostrally. Explant of ventral half of the DM was cocultured with the VM taken from the other half of the midbrain (inset). $\mathrm{TH}^{+}$axons extending from the $\mathrm{VM}$ change growth direction and extend following the polarity of the substratum. Scale bar, $100 \mu \mathrm{m}$.

1979). To address this issue, we cut the DM into rostral and caudal halves along the dorsoventral axis and (1) prepared double caudal halves, or (2) transposed them along the rostrocaudal axis, to coculture with VM explants. These manipulations should not affect the growth of $\mathrm{TH}^{+}$axons if a homogeneously distributed cue is responsible (Fig. 6A), whereas the axons should be unable to grow across the border of double caudal DM or transposed explants, if a gradient contributes to the guidance (Fig. 6B). Most $\mathrm{TH}^{+}$axons failed to grow across the border of two DM tissues in both double caudal (Fig. 6C,E) $(n=16)$ and transposed DM arrangements (Fig. $6 G)(n=11)$, whereas in control experiments in which DM was cut into two halves but cultured in their original position, $\mathrm{TH}^{+}$axons tended to advance ignoring the explant border (Fig. $6 D, F)(n=5)$. These results favor the possibility that some sort of gradient contributes to the guidance of $\mathrm{TH}^{+}$axons along the rostrocaudal axis.

\section{DISCUSSION}

\section{Development of dopaminergic axons in vivo and in vitro}

We have developed a culture preparation that can mimic the development and pathfinding of dopaminergic axons in vivo. Midbrain dopaminergic neurons are born at E12-E15 (Altman and Bayer 1981; Specht et al., 1981; Marchand and Poirier 1983) (see also König et al., 1988) and develop in the ventral midbrain by signals from the floor plate and the isthmus (Hynes et al., 1995a,b; Ye et al., 1998), projecting axons rostrally to innervate the striatum, limbic system, and neocortex (Lindall and Björklund, 1983). Consistent with the literature, the present immunostaining of rat whole-mount preparation demonstrated the presence of a substantial number of $\mathrm{TH}^{+}$neurons in the ventral midbrain of E12-E13 rat embryos. $\mathrm{TH}^{+}$axons departed from the ventral midline at E12 and extended rostrally, forming a bundle that appeared to course along a specific path. Although their trajectories were somewhat different from those in vivo in that a substantial number of axons deflected dorsally, most axons grew rostrally, indicating that the mechanisms that guide these axons rostrally are essentially preserved in our in vitro preparations.

\section{Local directional cues may guide dopaminergic axons rostrally}

The present study suggests that local directional cues contribute to the guidance of dopaminergic axons. This is in contrast to the guidance of circumferentially extending axons in which long-range diffusible cues play a pivotal role (Colamarino and TessierLavigne, 1995a). The present results do not preclude the possibility that some sort of gradient had been established in the midbrain region before the start of culture; some diffusible cues originating from the isthmus, for example, may form a gradient by binding to the extracellular matrix. However, occurrence of rostrally oriented growth of axons in ventral midbrain tissue that does not include the isthmus or the diencephalon (Figs. 3, 4) argues against the idea that freely diff usible cues from these regions are directly involved in the guidance of dopaminergic axons. Failure to observe a repellent activity of the isthmus toward $\mathrm{TH}^{+}$axons in collagen gel culture is consistent with this notion. Moreover, it seems unlikely that our culture preparation, which is not embedded in collagen gel, allows diffusion of molecules over long distances. The fact that $\mathrm{TH}^{+}$ axons grew rostrally even in a small piece of VM explant (Fig. 4D, arrowheads) further supports the notion that short-range cues in the midbrain direct these axons rostrally. Taken together, our results suggest directional cues localized to the midbrain substratum guide $\mathrm{TH}^{+}$axons rostrally.

In a number of attempts to uncover the polarity of the neural tube, brain or spinal cord tissue was rotated along the rostrocaudal axis in early stages of development. In these studies most longitudinally growing axons appeared to ignore the rotation (Hibbard, 1965; Holder et al., 1987; Yaginuma and Oppenheim, 1991; Matsuno and Nakamura, 1993; Nordlander and Liu, 1996). Although these results might indicate that rostrocaudal polarity contributing to axon guidance is absent in the neural tube, it is also possible that the transplanted brain or spinal cord was repolarized because of signals from host tissues. The arrangement and short culture period in our study may have minimized such an influence, if present, from neighboring tissues, allowing us to demonstrate rostrocaudal polarity of midbrain tissue.

\section{Nature of local directional cues}

Although staining of E12 preparations with an antibody against a-acetylated tubulin revealed the presence of longitudinally extending axons coursing around the alar/basal plate boundary (Y. Ito, R. Shirasaki, and F. Murakami, unpublished observation), it seems unlikely that $\mathrm{TH}^{+}$axons follow some "pioneering" axons that develop earlier, because the presence of these axons do not explain gradual rostral turning of $\mathrm{TH}^{+}$axons. Occurrence of rostrally oriented growth of $\mathrm{TH}^{+}$axons in a small piece of ventral midbrain tissue (Fig. 4D, $L V M$ ) also supports this notion.

The present results that $\mathrm{TH}^{+}$axons failed to extend across the border of two halves of DM are consistent with the idea that some sort of substratum-associated gradient contributes to the guidance of these axons. What is the nature of the presumptive gradient? In peripheral tissues of insects a gradient of epithelial cell adhesiveness along the proximal-distal axis was suggested to contribute to the guidance of sensory axons (Nardi, 1983; Caudy and Bentley, 1986). More recently a gradient of a repulsive molecule, semaphorin $2 \mathrm{a}$, has been shown to guide sensory axons centrally in the grasshopper limb bud (Isbister et al., 1999). Considering that $\mathrm{TH}^{+}$ neurons appear near the midbrain-hindbrain boundary, the isthmus, it would be an interesting possibility that molecules under the control of the organizing activity of the isthmus, such as ephrins A2 and A5 (Cheng et al., 1995; Drescher et al., 1995; Nakamoto et al., 1996; Frisén et al., 1998) (see also Logan et al., 1996; Shigetani et al., 1997; Drescher et al., 1997) control growth polarity of axons along the rostrocaudal axis by their repulsive activity. However, in zebrafish mutant, acerebellar, which is a mutant for $f g f 8$ and lacks 

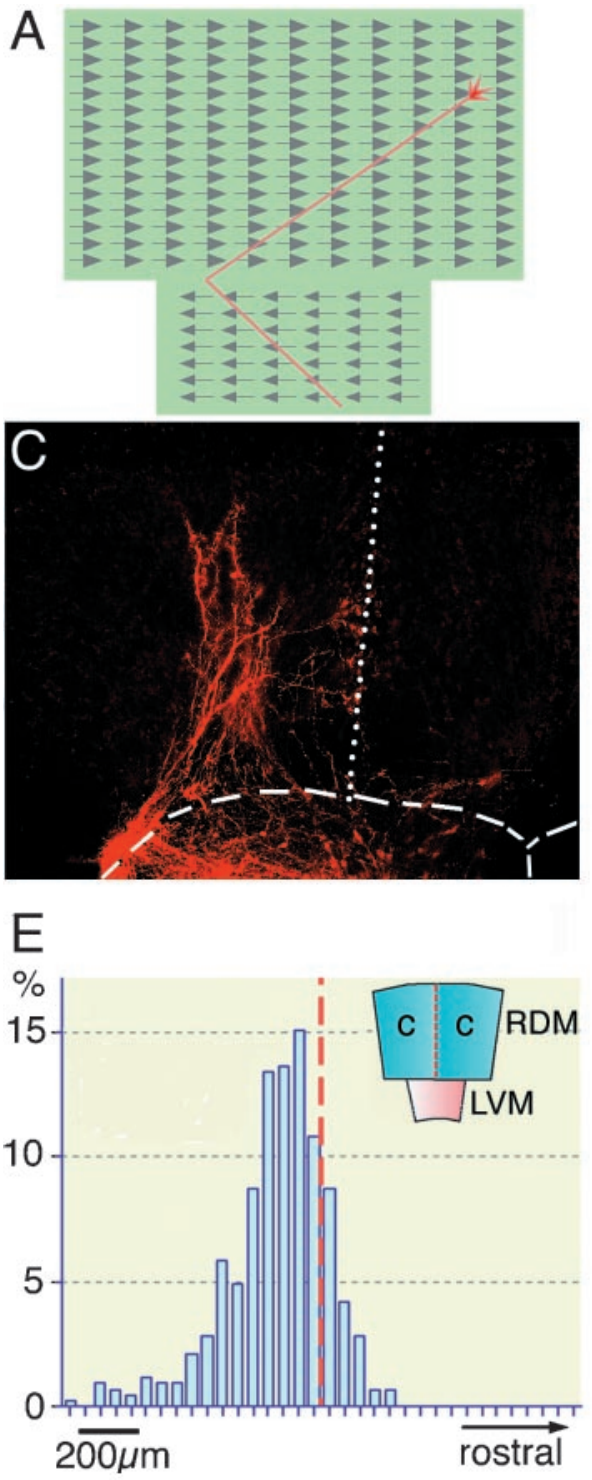

$\mathrm{G}$
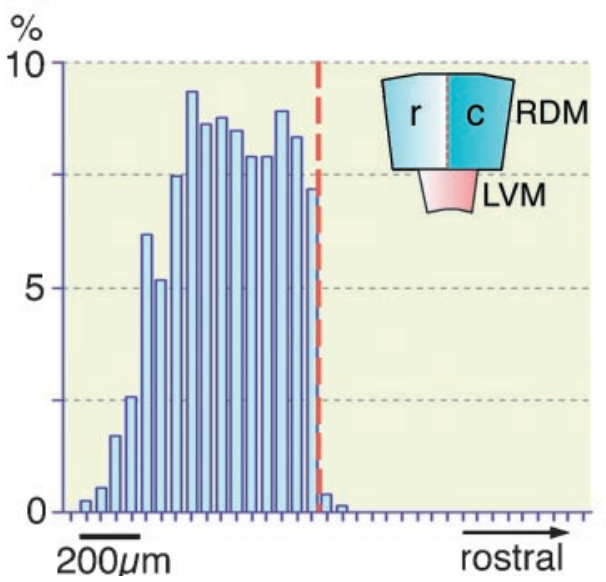
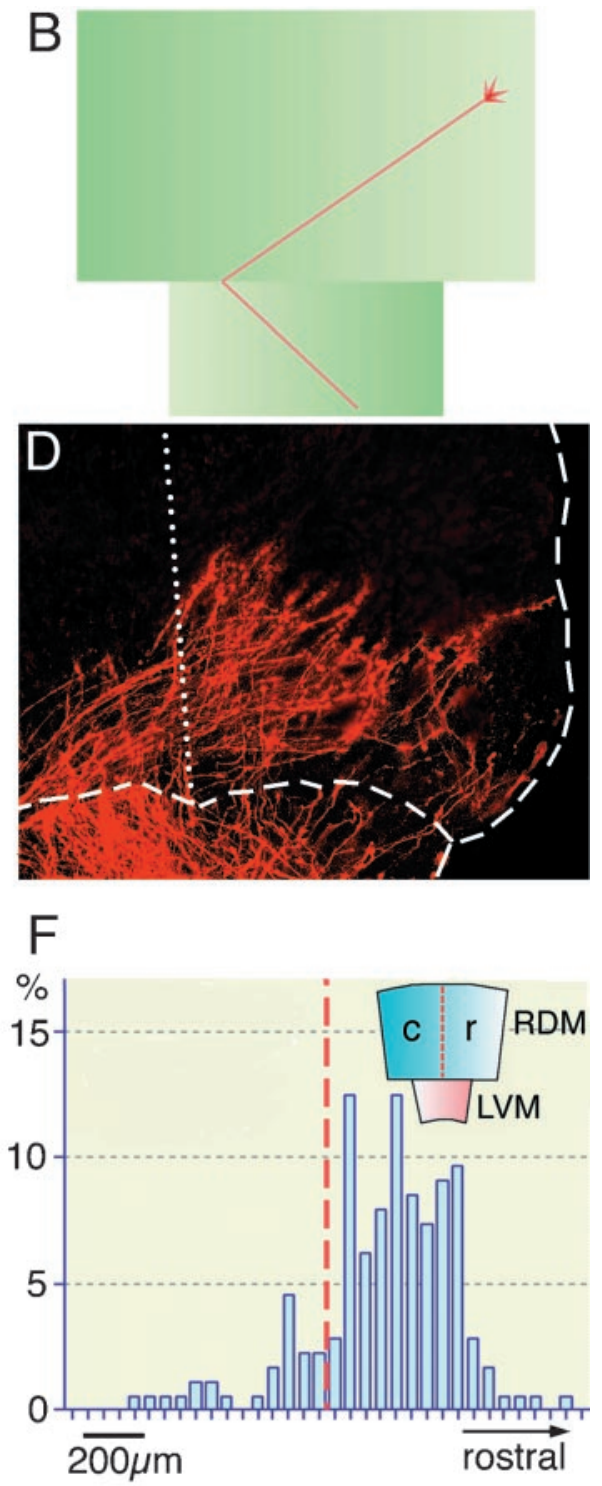
$\mathrm{H}$ expressed by the distance from the border between the two DM explants in culture with double caudal DM explants $(E)$, control culture $(F)$, and transposed DM explants $(G)$. Although many $\mathrm{TH}^{+}$axons stayed in the left half of the explants in both experiments, less axons tended to approach the border in $G$ compared to $E$. This suggests that the activity that causes the rostrally directed growth of $\mathrm{TH}^{+}$is stronger in the caudal than the rostral half. Insets in the top left corner of $E-G$ show the arrangement of explants. $H$, A diagram illustrating the method of measurement. $c$, Caudal; $r$, rostral. A total of 176 axons were analyzed from five control cultures, 425 axons from 16 cultures in which two caudal halves of DM were put together, and 696 axons from 11 cultures in which rostral and caudal halves were transposed. $V M$, Ventral mesencephalic explant; $D M$, dorsal mesencephalic explant.

preliminary experiments in which VM explants were cocultured the isthmus, rostrocaudal polarity of the retinotectal map was only partially disrupted (Picker et al., 1999), implying that molecules that are not under the control of the organizing activity of the isthmus play an important role in regulating growth polarity of midbrain axons along the rostrocaudal axis. Interestingly, in our juxtaposed to dorsal myelencephalon, $\mathrm{TH}^{+}$axons entering the dorsal myelencephalon explant changed their growth direction and extended rostrally. This observation is consistent with the notion that cues directing $\mathrm{TH}^{+}$axons rostrally do not peak at the isthmus.

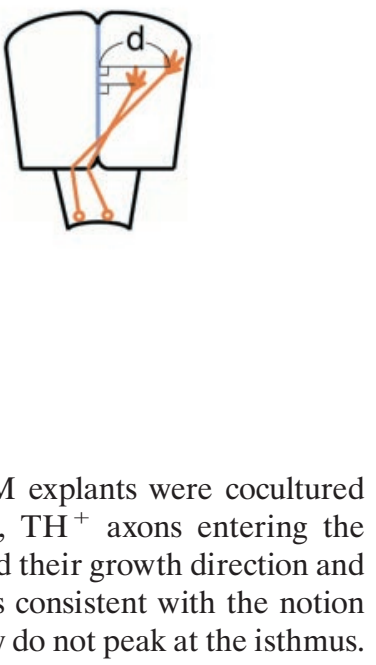




\section{Roles of local directional cue in other systems}

Retinal axons growing into a small piece of rotated transplant of presumptive optic tract of Xenopus often show severely disorganized growth (Harris, 1989), suggesting that a substrateassociated directional cue in the diencephalon may also be involved in the guidance of retinal axons. Other monoaminergic neurons, i.e., serotonergic and noradrenergic, originating from the hindbrain, also extend axons rostrally along the longitudinal axis, although a part of their axons extends caudally. Whether these neurons also use local directional cues for their navigation along the rostrocaudal axis awaits further studies.

In conclusion, the present study has provided unequivocal evidence that navigation of dopaminergic axons along the rostrocaudal axis in the vertebrate CNS is regulated by local directional cues. It would be an interesting possibility that long-range diff usible cues control the dorsoventral polarity, whereas short-range directional cues control rostrocaudal polarity of axonal growth.

\section{REFERENCES}

Altman J, Bayer SH (1981) Development of the brain stem in the rat. V. Thymidine-radiographic study of the time of origin of neurons in the midbrain tegmentum. J Comp Neurol 198:677-716.

Bally-Cuif L, Wassef M (1995) Determination events in the nervous system of the vertebrate embryo. Curr Opin Genet Dev 5:450-458.

Bentley D, Caudy M (1983) Navigational substrates for peripheral pioneer growth cones: limb-axis polarity cues, limb-segment boundaries, and guidepost neurons. Cold Spring Harb Symp Quant Biol 48:573-585.

Berlot J, Goodman CS (1984) Guidance of peripheral pioneer neurons in the grasshopper: adhesive hierarchy of epithelial and neuronal surfaces. Science 223:493-496.

Caudy M, Bentley D (1986) Pioneer growth cone morphologies reveal proximal increases in substrate affinity within leg segments of grasshopper embryos. J Neurosci 6:364-379.

Cheng HJ, Nakamoto M, Bergemann AD, Flanagan JG (1995) Complementary gradients in expression and binding of ELF-1 and Mek4 in development of the topographic retinotectal projection map. Cell 82:371-381.

Colamarino SA, Tessier-Lavigne M (1995a) The role of the floor plate in axon guidance. Annu Rev Neurosci 18:497-529.

Colamarino SA, Tessier-Lavigne M (1995b) The axonal chemoattractant netrin-1 is also a chemorepellent for trochlear motor axons. Cell 81:621-629.

Crossley PH, Martinez S, Martin GR (1996) Midbrain development induced by FGF8 in the chick embryo. Nature 380:66-68.

Drescher U, Kremoser C, Handwerker C, Löschinger J, Noda M, Bonhoeffer F (1995) In vitro guidance of retinal ganglion cell axons by RAGS, a $25 \mathrm{kDa}$ tectal protein related to ligands for Eph receptor tyrosine kinases. Cell 82:359-370.

Drescher U, Bonhoeffer F, Müller BK (1997) The Eph family in retinal axon guidance. Curr Opin Neurobiol 7:75-80.

Frisén J, Yates PA, McLaughlin T, Friedman GC, O'Leary DDM, Barbacid M (1998) Ephrin-A5 (AL-1/RAGS) is essential for proper retinal axon guidance and topographic mapping in the mammalian visual system. Neuron 20:235-243.

Guthrie S, Pini A (1995) Chemorepulsion of developing motor axons by the floor plate. Neuron 14:1117-1130.

Harris WA (1989) Local positional cues in the neuroepithelium guide retinal axons in embryonic Xenopus brain. Nature 339:218-221.

Hibbard E (1965) Orientation and directed growth of mauthner's cell axons from duplicated vestibular nerve roots. Exp Neurol 13:289-301.

Holder N, Clarke JDW, Tonge D (1987) Pathfinding by dorsal column axons in the spinal cord of the frog tadpole. Development 99:577-587.

Hynes M, Porter JA, Chiang C, Chang D, Tessier-Lavigne M, Beachy PA, Rosenthal A (1995a) Induction of midbrain dopaminergic neurons by sonic hedgehog. Neuron 15:35-44.

Hynes M, Poulsen K, Tessier-Lavigne M, Rosenthal A (1995b) Control of neuronal diversity by the floor plate: Contact-mediated induction of midbrain dopaminergic neurons. Cell 80:95-101.

Isbister CM, Tsai A, Wong ST, Kolodkin AL, O'Connor TP (1999)
Discrete roles for secreted and transmembrane semaphorins in neuronal growth cone guidance in vivo. Development 126:2007-2019.

Kelley OG, Melton A (1995) Induction and patterning of the vertebrate nervous system. Trends Genet 11:273-278.

König N, Wilkie MB, Lauder JM (1988) Tyrosine hydroxylase and serotonin containing cells in embryonic rat rhombencephalon: a wholemount immunocytochemical study. J Neurosci Res 20:212-223.

Lee SMK, Danielian PS, Fritzsch B, McMahon AP (1997) Evidence that FGF8 signalling from the midbrain-hindbrain junction regulates growth and polarity in the developing midbrain. Development 124:959-969.

Lindall O, Björklund A (1983) Dopamine- and norepinephrinecontaining neuron systems. Their anatomy in the rat brain. In: Chemical neuroanatomy (Emson PC, ed), pp 229-255. New York: Raven.

Logan C, Wizenmann A, Drescher U, Monschau B, Bonhoeffer F, Lumsden A (1996) Rostral optic tectum acquires caudal characteristics following ectopic engrailed expression. Curr Biol 6:1006-1014.

Lumsden A, Krumlauf R (1996) Patterning the vertebrate neuraxis. Science 274:1109-1115.

Marchand R, Poirier LJ (1983) Isthmic origin of neurons of the rat substantia nigra. Neuroscience 9:373-381.

Matsuno T, Nakamura H (1993) Plasticity of avian mesencephalic polarity revealed by trajectories of tectofugal axons. Dev Brain Res 75:39-44.

Murakami F, Shirasaki R (1997) Guidance of circumferentially growing axons by the floor plate in the vertebrate central nervous system. Cell Tissue Res 290:323-330.

Nakamoto M, Cheng HJ, Friedman GC, McLaughlin T, Hansen MJ, Yoon CH, O'Leary DD, Flanagan JG (1996) Topographically specific effects of ELF-1 on retinal axon guidance in vitro and retinal axon mapping in vivo. Cell 86:755-766.

Nardi JB (1983) Neuronal pathfinding in developing wings of the moth Manduca sexta. Dev Biol 95:163-174.

Nordlander RH, Liu S (1996) Axons of Xenopus neural tube respond to reversals of neural tube orientation. J Neurobiol 29:490-502.

Palka J (1979) Theories of pattern formation in insect neural development. Adv Insect Physiol 14:251-349.

Picker A, Brennan C, Reifers F, Clarke JDW, Holder N, Brand M (1999) Requirement for the zebrafish mid-hindbrain boundary in midbrain polarisation, mapping and confinement of the retinotectal projection. Development 126:2967-2978.

Ramón y Cajal S (1892) La rétine des vertébres. La Cellule 9:119-258.

Shigetani Y, Funahashi J, Nakamura H (1997) En-2 regulates the expression of the ligands for Eph type tyrosine kinases in chick embryonic tectum. Neurosci Res 27:211-217.

Shirasaki R, Tamada A, Katsumata R, Murakami F (1995) Guidance of cerebellofugal axons in the rat embryo: directed growth toward the floor plate and subsequent elongation along the longitudinal axis. Neuron 14:961-972.

Shirasaki R, Mirzayan C, Tessier-Lavigne M, Murakami F (1996) Guidance of circumferentially growing axons by netrin-dependent and -independent floor plate chemotropism in the vertebrate brain. Neuron 17:1079-1088.

Shirasaki R, Katsumata R, Murakami F (1998) Change in chemoattractant responsiveness of developing axons at an intermediate target. Science 279:105-107.

Specht LA, Pickel VM, Joh TH, Reis DJ (1981) Light-microscopic immunocytochemical localization of tyrosine hydroxylase in prenatal rat brain. I. Early ontogeny. J Comp Neurol 199:233-253.

Tamada A, Shirasaki R, Murakami F (1995) Floor plate chemoattracts crossed axons and chemorepels uncrossed axons in the vertebrate brain. Neuron 14:1083-1093.

Tessier-Lavigne M (1992) Axon guidance by molecular gradients. Curr Opin Neurobiol 2:60-65.

Tessier-Lavigne M, Placzek M, Lumsden A, Dodd J, Jessell TM (1988) Chemotropic guidance of developing axons in the mammalian central nervous system. Nature 336:775-778.

Yaginuma H, Oppenheim RW (1991) An experimental analysis of in vivo guidance cues used by axons of spinal interneurons in the chick embryo: evidence for chemotropism and related guidance mechanisms. J Neurosci 11:2598-2613.

Yamamoto N, Kurotani T, Toyama K (1989) Neural connections between the lateral geniculate nucleus and visual cortex in vitro. Science 245:192-194.

Ye W, Shimamura K, Rubenstein JLR, Hynes MA, Rosenthal A (1998) FGF and Shh signals control dopaminergic and serotonergic cell fate in the anterior neural plate. Cell 93:755-766. 